\title{
ERRATA, VOLUME 74
}
A. Devinatz, Integral representations of positive definite functions.
p. 64 , line 9. For " $i s_{r} \cdot u_{k}$ " read " $i s_{r}^{(k)} u_{k}$," in two places.
p. 73, line 2. For " $+y \cdot u_{k}$ " read " $-y^{(k)} u_{k}$ " 